https://doi.org/10.53937/icz10.2021.61

\title{
USING IN SILICO RFLP METHOD FOR THE STUDY OF MC1R GENE ALLELES IN THE SPECIES SUS SCROFA
}

\author{
Victor Sitnic
}

Institute of Zoology, Chisinau, Republic of Moldova, e-mail: sitnic.md@gmail.com

Abstract. In the present study we aimed to use a bioinformatics algorithm that predict RFLP fragmentation of swine MC1R alleles simulating the sequence digestion with over 700 restriction enzymes. The results show several restriction enzymes that have the potential to be used for genotyping of Sus scrofa individuals and for differentiation between hybrids and pure line wild boars (WB)/domestic pigs (DP). Genotyping studies of wild boars and domestic pigs in Moldova populations would allow the determination of introgression/backcrossing degree and would contribute to the elaboration of adequate conservation measures

\section{Introduction}

The $M C 1 R$ is a gene that regulates melanin synthesis and in some animals plays a central role in the pigmentation process. It contains instructions for producing the protein Melanocortin 1 Receptor which is located in the melanocyte membrane. Sequencing analyzes showed that species Sus scrofa has several alleles of this gene. The multiple allelism of the MC1R gene can be observed phenotypically through the diversity of skin pigmentation in pigs. The wild-type allele named $\mathrm{E}^{+}$has been identified in wild boars, $\mathrm{E}^{\mathrm{D} 1}$ allele - in domestic breeds Large Black and Meishan, $\mathrm{E}^{\mathrm{PD} 2}$ in Hampshire, Large White and Pietrain breeds [1,5], while recessive allele e is associated with the breed Duroc (Fig.1).

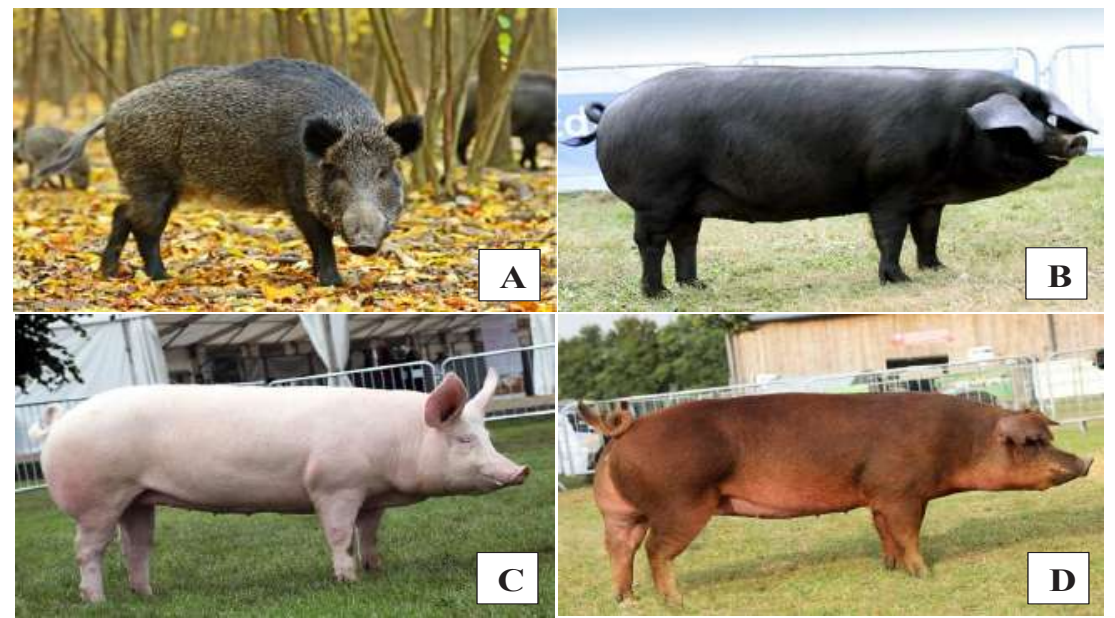

Figure 1. Phenotypic skin color traits in the species Sus scrofa with different MC1R alleles (A - Wild Boar [3], B - Large Black [4], C - Large White [4], D - Duroc [4])

The wild boar is the ancestor of the domestic pig and can successfully crossbreed with it. This can happen unintentionally in areas of open or semi-open swine farms or by intentional crossing. WB populations have been increasing worldwide, and in many countries it is con- 
sidered a forest and agricultural pest [10]. A recent hypothesis suggests that introgressive hybridization between wild boar and domestic pig increases WB invasiveness [2]. Also, there are concerns that hybrids between wild and domestic animals may affect the genetic integrity and wild adaptive potential of WB. Currently, the degree to which WB and DP hybridization occurs in the Republic of Moldova is unclear.

Sequencing of the $M C 1 R$ swine gene would be an extremely effective way for pig genotyping, however, even today it is not very accessible and remains an expensive molecular method. This makes necessary the use of cheaper alternatives for identification of pig hybrids. One of these alternatives would be the discrimination between sequences of interest by using Restriction Fragment Length Polymorphism (RFLP). In the present study we aimed to use a bioinformatics algorithm that predict RFLP fragmentation of swine $M C 1 R$ alleles simulating the sequence digestion with over 700 restriction enzymes.

\section{Materials and methods}

In the species Sus scrofa, the MC1R gene has 963 base pairs and wild-type allele (E+) is fully expressed in pure line wild boars. Other alleles differ by changes in the following positions $[1,5]$ :

1. c.370G $>\mathrm{A}$ - Hampshire, Large White and Pietrain breeds (EPD2)

2. c. $283 \mathrm{G}>\mathrm{A}$, c. $305 \mathrm{~T}>\mathrm{C}$ and c.363T $>\mathrm{C}$ - Large Black and Meishan (ED1)

3. c. $491 \mathrm{C}>\mathrm{T}$ and c. $727 \mathrm{G}>\mathrm{A}-$ Duroc breed (e)

The wild-type MC1R sequence has been extracted in FASTA format from NCBI [6], and alleles alignment has been performed in R language [9] using DECIPHER [11] and Biostrings [7] packages (Fig.2).

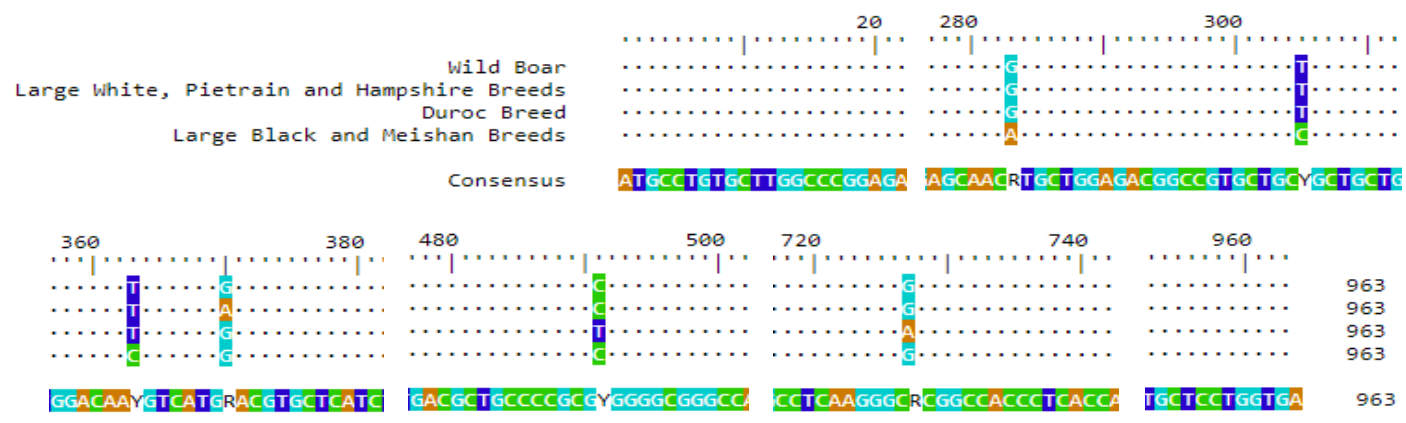

Figure 2. Swine MC1R alleles alignment

Simulation and visualization of Restriction Fragment Length Polymorphism (RFLP) patterns from $D N A$ sequences were accomplished utilizing seqRFLP package [8] in R. This package includes functions for handling $D N A$ sequences, especially for simulating RFLP patterns based on selected restriction enzymes and $D N A$ sequences. 


\section{Results and discussions}

In silico digestion of the studied sequences allowed the identification of several restriction enzymes that have the potential to be used for genotyping of Sus scrofa individuals and for differentiation between hybrids and pure line WB/DP. In Fig.3 is shown the simulated electrophoregram obtained after virtual digestion of studied allelic sequences with the next restriction enzymes: AjiI, BspHI, BstUI, NspI, FnuDII, MhlI, PcsI, TspDTI.

The enzymes shown in fig. 3 were selected following individual in silico analyzes of over 700 candidates and they can discriminate between sequences as follows:

1. AjiI, BspHI and TspDTI - distinguish among $\mathrm{E}^{\mathrm{PD} 2}$ and other alleles

2. BstUI, FnuDII and MhlI - distinguish among e and other alleles

3. NspI and PcsI - distinguish among $\mathrm{E}^{\mathrm{D} 1}$ and other alleles

Theoretically, it is expected that in the Republic of Moldova the predominant allele in pig farms is $E^{\mathrm{PD} 2}$, therefore the enzymes from the first category that could discriminate between this domestic allele and E+ wild-type are of increased interest. AjiI (BmgBI) candidate recognizes $C A C^{\wedge} G T C$ sequences while $B s p H I$ cuts at $T^{\wedge} C A T G A$ sites, both enzymes having an optimum temperature of $37^{\circ} \mathrm{C}$. Although $T_{s} p D T I$ discriminates by producing two electrophoretic bands in $\mathrm{E}+$ allele and three in $\mathrm{E}^{\mathrm{PD} 2}$, it is more difficult to be found, that's why the first two candidates seem to be more practical. The pure lines of Large Black, Meishan and Duroc breeds are probably less common in Moldova, however, the enzymes from categories 2 and 3 present an interest for determination of possible heterozygotes, carriers of $\mathrm{E}^{\mathrm{D} 1}$ and e alleles. Other restriction enzymes that may be useful for differentiating MCIR variants are BdaI, BstNSI, BtrI, CciI, FatI, PagI, RcaI, SduI, XceI.

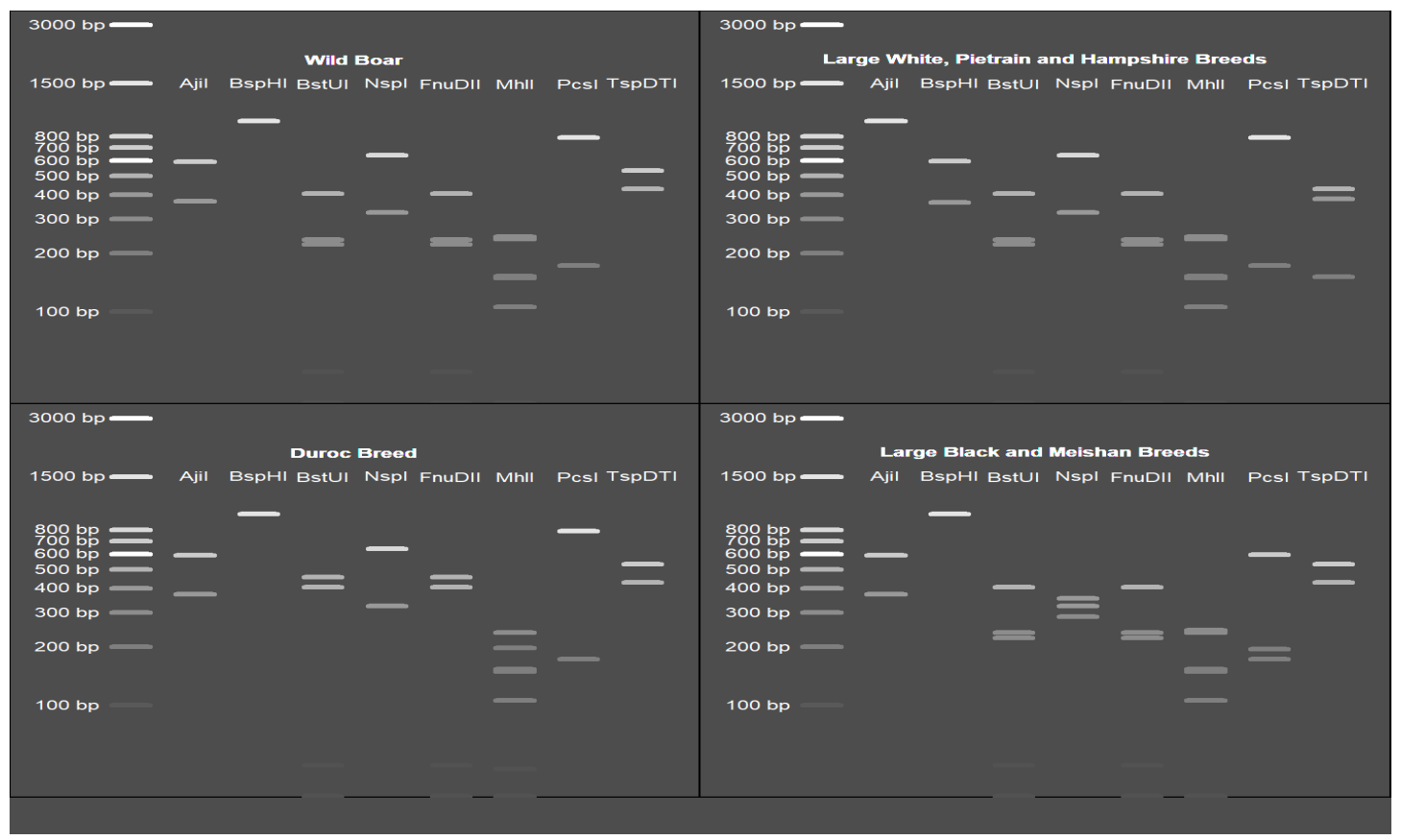

Figure 3. Simulated electrophoregram obtained after virtual restriction enzymes (AjiI, BspHI, BstUI, NspI, FnuDII, MhII, PcsI, TspDTI) digestion of Sus scrofa allelic sequences 
In the current study we used $M C 1 R$ gene polymorphism for identification of swine genotyping possibilities with in silico RFLP method. Good results for differentiation between hybrids and pure lines could be achieved only by correct enzymes selection and by their combination. The combination would increase the chance of a correct result and would allow the identification of heterozygous individuals. Genotyping studies of wild boars and domestic pigs in Moldova populations would allow the determination of introgression/backcrossing degree and would contribute to the elaboration of adequate conservation measures. We recommend the wet laboratory testing of the above mentioned enzymes and the in vitro evaluation of their genotyping potential.

The study has been conducted within the State Program project 20.80009.7007.02.

\section{Bibliography}

1. Dzialuk, A., Zastempowska, E., Skórzewski, R. et al. High domestic pig contribution to the local gene pool of free-living European wild boar: a case study in Poland. Mamm Res 63, 65-71 (2018). https://doi.org/10.1007/s13364-017-0331-3

2. Frantz AC, Zachos FE, Kirschning J et al (2013) Genetic evidence for introgression between domestic pigs and wild boars (Sus scrofa) in Belgium and Luxembourg: a comparative approach with multiple marker systems. Biol J Linn Soc 110:104-115. doi:10.1111/bij.12111

3. https://www.alimentarium.org/en/knowledge/wild-boar

4. https://www.britishpigs.org.uk/

5. Kijas JM, Wales R, Törnsten A, Chardon P, Moller M, Andersson L. Melanocortin receptor 1 (MC1R) mutations and coat color in pigs. Genetics. 1998 Nov;150(3):1177-85. PMID: 9799269; PMCID: PMC1460407.

6. Maselli,V. and Fulgione,D. Submitted (20-MAY-2015) Department of Biology, University of Naples Federico II, Campus Monte S. Angelo, Naples, Campania 80126, Italy. https://www. ncbi.nlm.nih.gov/nuccore/KR865958.1?report=fasta

7. Pagès H., Aboyoun P., Gentleman R., DebRoy S. Biostrings: Efficient manipulation of biological strings. R package version 2.52.0., 2019.

8. Qiong Ding and Jinlong Zhang (2012). seqRFLP: Simulation and visualization of restriction enzyme cutting pattern from DNA sequences. R package version 1.0.1. https://CRAN.R-project.org $/$ package $=$ seqRFLP.

9. R Core Team (2020). R: A language and environment for statistical computing. R Foundation for Statistical Computing, Vienna, Austria. URL https://www.R-project.org/.

10. Wilson CJ (2013) The establishment and distribution of feral wild boar (Sus scrofa) in England. Wildl Biol Pract. doi:10.2461/wbp.2014.un.1

11. Wright E. S. Using DECIPHER v2.0 to Analyze Big Biological Sequence Data in R. The R Journal, (2016). No 8(1), p.352-359. 